\title{
The CRAN bayesvl package on U-Toronto Stats
}

The Comprehensive R Archive Network (CRAN)

August 25, 2019

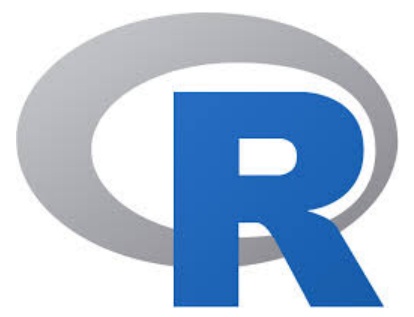

The R Core Team

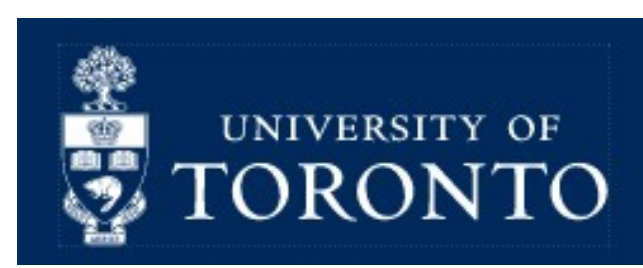

Department of Statistical Sciences

https://utstat.toronto.edu/cran/web/packages/bayesvl/index.html 
bayesvl: Visually Learning the Graphical Structure of Bayesian Networks and Performing MCMC with 'Stan'

Provides users with its associated functions for pedagogical purposes in visually learning Bayesian networks and Markov chain Monte Carlo (MCMC) computations. It enables users to: a) Create and examine the (starting) graphical structure of Bayesian networks; b) Create random Bayesian networks using a dataset with customized constraints; c) Generate 'Stan' code for structures of Bayesian networks for sampling the data and learning parameters; d) Plot the network graphs; e) Perform Markov chain Monte Carlo computations and produce graphs for posteriors checks. The package refers to one reference item, which describes the methods and algorithms: Vuong, Quan-Hoang and La, Viet-Phuong (2019) < doi:10.31219/osf.io/w5dx6 $>$ The 'bayesvl' R package. Open Science Framework (May 18).

Version: $\quad 0.8 .5$

Depends: $\quad \mathrm{R}(\geq 3.4 .0)$, $\underline{\text { stan }}(\geq 2.10 .0)$, $\underline{\text { StanHeaders }}(\geq 2.18 .0)$, stats, graphics, methods

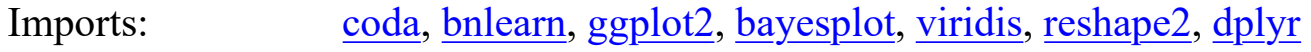

Suggests: $\quad \underline{\text { loo }}(\geq 2.0 .0)$

Published: $\quad$ 2019-05-24

Author: $\quad$ Viet-Phuong La [aut, cre], Quan-Hoang Vuong [aut]

Maintainer: $\quad$ Viet-Phuong La $<$ lvphuong at gmail.com>

BugReports: $\quad$ https://github.com/sshpa/bayesvl/issues

License: $\quad \underline{\text { GPL }}(\geq 3)$.

URL: $\quad$ https://github.com/sshpa/bayesvl

NeedsCompilation: no

CRAN checks: $\quad$ bayesvl results

Downloads :

Reference manual: bayesvl.pdf

Package source: bayesvl 0.8.5.tar.gz

Windows binaries: r-devel: bayesvl 0.8.5.zip, r-release: bayesvl 0.8.5.zip, r-oldrel: bayesvl_0.8.5.zip

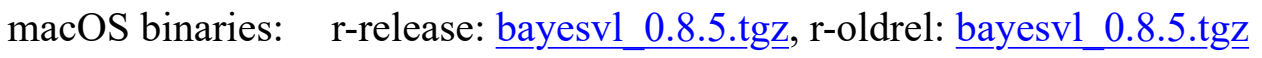

Linking :

Please use the canonical form https://CRAN.R-project. org/package=bayesvl to link to this page. 


\section{References}

La, V. P., \& Vuong, Q. H. (2019). bayesvl: Visually learning the graphical structure of Bayesian networks and performing MCMC with 'Stan'. The Comprehensive R Archive Network (CRAN). Available online: https://cran.r-project.org/package=bayesvl

Vuong, Q. H., \& La, V. P. (2019). BayesVL package for Bayesian statistical analyses in R. GitHub. Available online: https://github.com/sshpa/bayesvl

Vuong, Q. H., La, V. P., Nguyen, M. H., Ho, M. T., Ho, M. T., \& Mantello, P. (2020). Improving Bayesian statistics understanding in the age of Big Data with the bayesvl R package. Software Impacts, 4, 100016.

Vuong, Q. H., La, V. P., Nguyen, M. H., Ho, M. T., Tran, T., \& Ho, M. T. (2020). Bayesian analysis for social data: A step-by-step protocol and interpretation. MethodsX, 7, 100924. 